\title{
Algebraically diverging modes upstream of a swept bluff body
}

\author{
Dominik Obrist ${ }^{1} \dagger$ and Peter J. Schmid ${ }^{2}$ \\ ${ }^{1}$ Institute of Fluid Dynamics, ETH Zurich, 8092 Zürich, Switzerland \\ ${ }^{2}$ Laboratoire d'Hydrodynamique (LadHyX), CNRS-École Polytechnique, 91128 Palaiseau, France
}

(Received 16 February 2011; revised 5 May 2011; accepted 13 June 2011; first published online 2 August 2011)

Classical stability theory for swept leading-edge boundary layers predicts eigenmodes in the free stream with algebraic decay far from the leading edge. In this article, we extend the classical base flow solution by Hiemenz to a uniformly valid solution for the flow upstream of a bluff body, which includes a three-dimensional boundary layer, an inviscid stagnation-point flow and an outer parallel flow. This extended, uniformly valid base flow additionally supports modes which diverge algebraically outside the boundary layer. The theory of wave packet pseudomodes is employed to derive analytical results for the growth rates and for the eigenvalue spectra of this type of mode. The complete spectral analysis of the flow, including the algebraically diverging modes, will give a more appropriate basis for receptivity studies and will more accurately describe the interaction of perturbations in the free stream with disturbances in the boundary layer.

Key words: boundary layer receptivity, boundary layer stability, instability

\section{Introduction and background}

The flow over a bluff body which is yawed at a prescribed angle against a uniform stream is a situation that commonly occurs, e.g. in aeronautical, marine and terrestrial vehicle aerodynamics. It has long been recognized that, in particular, the flow in the vicinity of the attachment line plays a crucial role, since both experiments and simulations have established that the perturbation dynamic near the attachment line strongly affects the development of disturbances further downstream. Consequently, the stability of swept attachment-line flow has attracted a great deal of interest and scientific effort.

The swept Hiemenz boundary layer (Hiemenz 1911) is a widely accepted model for the base flow close to the attachment line along a swept bluff body (figure 1). After assuming an explicit linear chordwise dependence only of the chordwise velocity component, $\boldsymbol{U}=(x U / \operatorname{Re}, V / R e, W)$, the three-dimensional base flow profile is given as a similarity solution for the normal velocity component $V$. The Reynolds number $R e$ is based on the free-stream sweep velocity $W_{\infty}$ and on a boundary-layer length scale which is given by the square root of the ratio between the kinematic viscosity and the shear rate of the stagnation-point flow in the free stream. The basic linear stability theory for this particular flow configuration was established in the work of Hall, Malik \& Poll (1984). Their modal perturbations follow the ansatz for the base flow, 


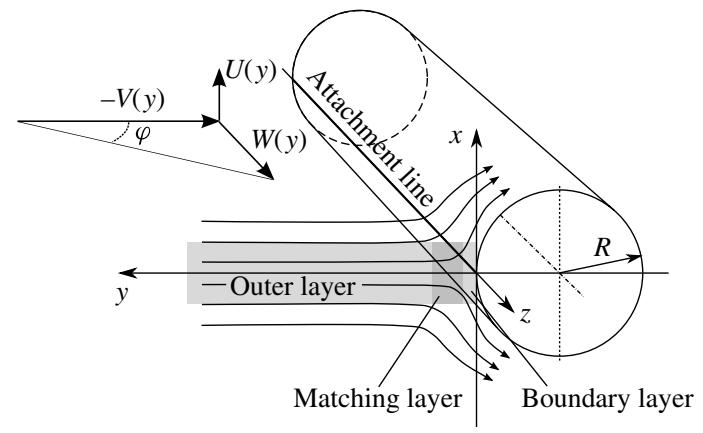

FIGURE 1. Flow around a swept cylinder with radius $R$ and sweep angle $\varphi$. The $x$-axis is oriented in the chordwise direction, the wall-normal $y$-axis points in the upstream direction, and the spanwise $z$-axis coincides with the attachment line.

i.e. $\boldsymbol{u}=(x \hat{u}(y), \hat{v}(y), \hat{w}(y))$, which had previously been employed by Görtler (1955) and Hämmerlin (1955) for plane Hiemenz flow.

The stability of modes with a higher polynomial order in the chordwise direction, and an exponential behaviour in the spanwise direction $z$ and time, according to

$$
(u, v, w)=\left(p_{N}(x) \hat{u}(y), p_{N-1}(x) \hat{v}(y), p_{N-1}(x) \hat{w}(y)\right) \times \exp [\mathrm{i}(\gamma z-\lambda t)]
$$

where $p_{N}(x)$ denotes a polynomial of order $N$, has been determined (see e.g. Obrist \& Schmid 2003) by solving the associated generalized eigenvalue problem

$$
\begin{gathered}
{\left[\partial_{y}^{2}-V \partial_{y}-\gamma^{2}+(N+1) V^{\prime}-\mathrm{i} \gamma \operatorname{Re} W\right] \hat{u}+V^{\prime \prime} \hat{v}=-\mathrm{i} \operatorname{Re} \lambda \hat{u},} \\
{\left[\left(\partial_{y}^{2}-V \partial_{y}-\gamma^{2}+(N-2) V^{\prime}-\mathrm{i} \gamma \operatorname{Re} W\right)\left(\partial_{y}^{2}-\gamma^{2}\right)-V^{\prime \prime} \partial_{y}-N V^{\prime \prime \prime}+\mathrm{i} \gamma \operatorname{Re} W^{\prime \prime}\right] \hat{v}} \\
-2 N\left(V^{\prime \prime}+V^{\prime} \partial_{y}\right) \hat{u}=-\mathrm{i} \operatorname{Re} \lambda\left(\partial_{y}^{2}-\gamma^{2}\right) \hat{v} .
\end{gathered}
$$

The real spanwise wavenumber is denoted by $\gamma$; the temporal frequency and growth rate are given by the complex eigenvalue $\lambda$.

It can be shown that the swept Hiemenz boundary layer supports eigenmodes which decay superexponentially outside the boundary layer like $\exp \left(-y^{2} / 2\right)$. For a second class of eigenmodes, most of the energy resides in the free-stream region, where the Hiemenz flow approaches the solution for an inviscid swept stagnation-point flow. In this regime, the modes exhibit two types of algebraic behaviour as $y$ tends to infinity: (i) in the algebraic modes of type A the wall-normal velocity component dominates, i.e. $\hat{v} \gg \hat{u}$, and behaves like $\hat{v} \sim y^{-v-4}$ where $v=-\left[N+2+\gamma^{2}+\mathrm{i} \operatorname{Re}(\gamma-\lambda)\right]$; (ii) in the modes of type B the chordwise velocity component dominates $(\hat{u} \gg \hat{v})$ and behaves like $\hat{u} \sim y^{-v-1}$. In order to satisfy the homogeneous boundary conditions at $y \rightarrow \infty$, the algebraic modes are required to decay. Thus, the imaginary part of the temporal eigenvalues $\lambda=\lambda_{r}+\mathrm{i} \lambda_{i}$ must satisfy

$$
\begin{aligned}
& \text { type A: } \lambda_{i}<-\left(N-2+\gamma^{2}\right) / R e, \\
& \text { type B: } \lambda_{i}<-\left(N+1+\gamma^{2}\right) / R e .
\end{aligned}
$$

The above conditions on the decay of the algebraic modes are based on the assumption that the Hiemenz solution is valid everywhere. However, this cannot be the case simply because the wall-normal velocity for Hiemenz flow grows beyond all bounds for $y \rightarrow$ $\infty$. As a consequence, the boundedness requirements (1.3) are misleading. 
We will show in this article that realistic flow configurations with a swept attachment boundary layer have a more complex flow field in the free stream than described by the Hiemenz flow alone. We will find that the conditions (1.3) are too stringent, and that eigensolutions which diverge algebraically in the region of the inviscid stagnation-point flow can readily be accommodated. It is interesting to point out that algebraically diverging modes had already been predicted by Dhanak \& Stuart (1995) for stagnation-point flow without sweep. Their finding has so far been little appreciated.

\section{A uniformly valid base-flow solution}

For the present study, we use a representative flow configuration with a swept cylinder of radius $R$ (figure 1 ) for which the potential flow solution is known. We will concentrate on the flow field upstream of the attachment line, i.e. the flow field in a narrow strip defined by $y>0$ and $|x| \ll R$. Nonetheless, the results below should also apply to more general bluff-body configurations.

We divide the flow field upstream of a swept cylinder into three layers: a parallel flow (outer layer), a swept stagnation-point flow (matching layer) and a three-dimensional boundary layer (inner layer). The potential flow around a swept cylinder of radius $R$ is given by

$$
\begin{aligned}
& U_{P}=\frac{2}{\tan \varphi} \frac{R^{2} x(y+R)}{\left[x^{2}+(y+R)^{2}\right]^{2}}, \\
& V_{P}=-\frac{1}{\tan \varphi}\left\{1+R^{2} \frac{x^{2}-(y+R)^{2}}{\left[x^{2}+(y+R)^{2}\right]^{2}}\right\} \text {, } \\
& W_{P}=1 \text {, }
\end{aligned}
$$

with $\varphi$ as the sweep angle of the cylinder (see figure 1). This solution is non-dimensionalized with the same reference values as the swept Hiemenz flow. Within the narrow strip of interest $(y>0$ and $|x| \ll R)$, the flow simplifies to

$$
U_{P}=\frac{2}{\tan \varphi} \frac{R^{2} x}{(y+R)^{3}}, \quad V_{P}=-\frac{1}{\tan \varphi}\left\{1-\frac{R^{2}}{(y+R)^{2}}\right\}, \quad W_{P}=1 .
$$

For $y \ll R$, this potential flow solution approaches asymptotically the swept stagnationpoint flow $\boldsymbol{U}=(2 x / R \tan \varphi,-2 y / R \tan \varphi, 1)$.

For $y \gg 1$, the swept Hiemenz boundary layer approaches the swept stagnation-point flow $\boldsymbol{U}=(x / \operatorname{Re},(-y+d) / \operatorname{Re}, 1)$, where $d \approx 0.65$ denotes the displacement thickness. If we choose $R e=R \tan \varphi / 2$ and replace $y$ by $y-d$ in (2.2), we can match the potential flow solution asymptotically to the Hiemenz boundary layer. For sufficiently large Reynolds numbers, this yields a uniformly valid base-flow solution, $\boldsymbol{U}=$ $(x U(y) / R e, V(y) / R e, W(y))$, within a domain bounded by $y>0$ and $|x| \ll R e$. The velocity profile for the wall-normal component $V(y)$ of this uniform solution is shown in figure 2.

\section{Algebraically diverging modes}

If we insert the new base flow into the stability (1.2), we can find algebraically diverging modes which satisfy the homogeneous boundary conditions for $y \rightarrow \infty$. Figure 3 shows three representative eigenmodes for $N=0$. The remaining parameters for this numerical example (and all the following examples) are $R e=1000, \gamma=0.3$ and $\varphi=45^{\circ}$. Two of the displayed modes exhibit algebraic growth within the matching 


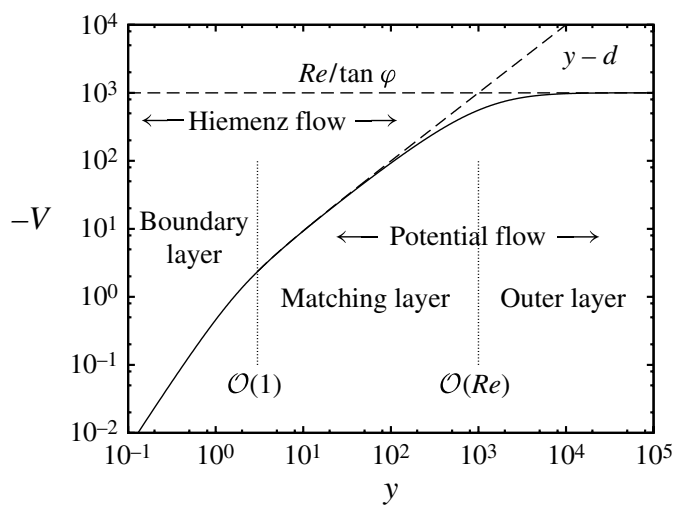

FIGURE 2. Wall-normal component $V(y)$ of the uniformly valid base-flow solution around a swept cylinder for $R e=1000$ and $\varphi=45^{\circ}$. The stagnation-point flow in the matching layer and the oncoming parallel flow in the outer layer are indicated with dashed lines.
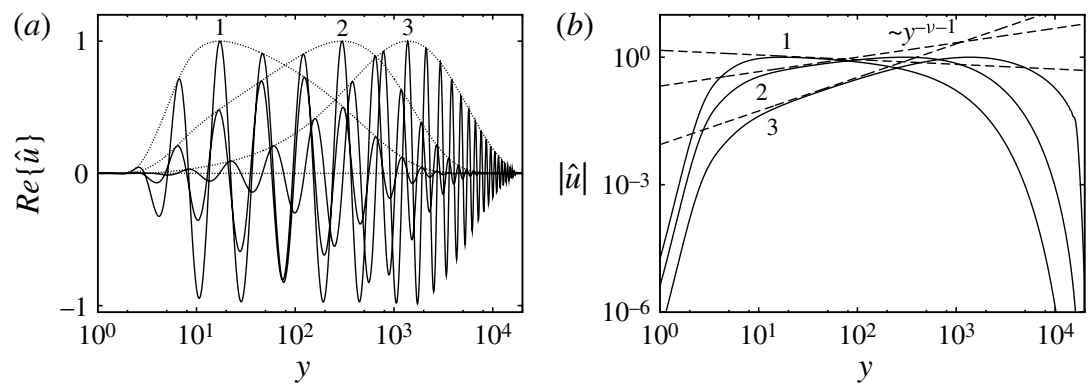

FIGURE 3. Eigenmodes for $N=0$. The corresponding eigenvalues are: mode $1, \lambda=(1.02-$ $0.4 \mathrm{i}) \gamma$; mode $2, \lambda=(1.02-0.25 \mathrm{i}) \gamma$; mode $3, \lambda=(1.02-0.1 \mathrm{i}) \gamma$. Modes 2 and 3 exhibit algebraic growth. (a) Real part of the eigenmodes; $(b)$ absolute values in a logarithmic scale; the theoretical algebraic decay/growth derived from the classical swept Hiemenz problem is indicated by dashed lines.

region $1 \ll y \ll R e$ but rapidly decay to zero as the stagnation-point flow blends into a parallel flow. Algebraically diverging eigenfunctions can also be found for higher-order modes (e.g. figure 4 for $N=3$ ). The eigenvalues associated with such modes do not satisfy the boundedness conditions (1.3); still, we will show that there does exist an upper limit for the imaginary part of the eigenvalues as well as for the algebraic growth rates of the corresponding eigenmodes.

\subsection{Wave packet pseudomodes}

We use the theory of wave packet pseudomodes (Trefethen 2005) to derive bounds for the eigenvalues of the algebraically diverging modes. This theory is described in detail by Trefethen \& Embree (2005) for ordinary differential equations. A wave packet pseudomode is an asymptotically accurate wave packet solution to an eigenvalue problem. Assuming a wave packet pseudomode localized in physical space around $y=y_{*}$ and localized in spectral space about a normal wavenumber $\beta=\beta_{*} / \epsilon$ (with $\epsilon$ as a small parameter) we can obtain, upon substitution of this ansatz into the generalized eigenvalue problem, an approximate eigenvalue $\lambda$ associated with this wave packet 

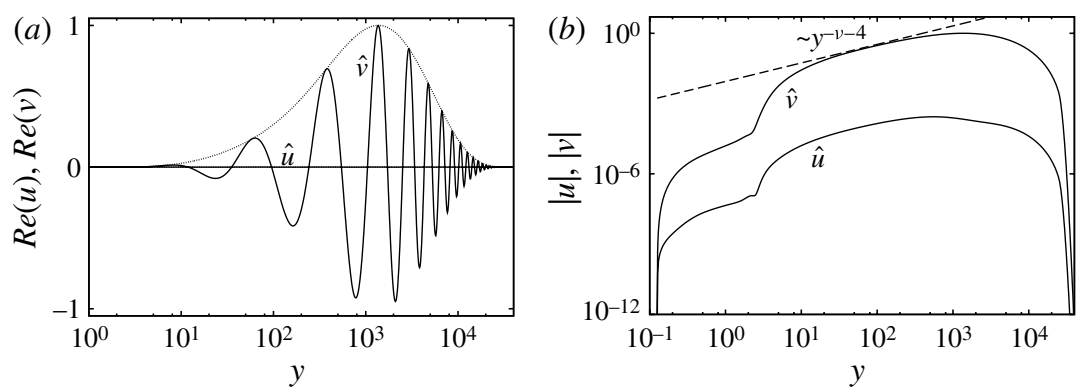

FIGURE 4. Algebraically diverging eigenmode for $N=3$ with the eigenvalue $\lambda=(1.01-$ $0.001 i) \gamma$. (a) Real part of the eigenmode; $(b)$ absolute values in a logarithmic scale; the theoretical algebraic growth derived from the classical swept Hiemenz problem is indicated by the dashed line.

pseudomode; it is given by the symbol $f\left(y_{*}, \beta_{*}\right)$ of the eigenvalue problem and is parametrized by $y_{*}$ and $\beta_{*}$. Formally, the symbol $f$ is an intermediate result of a Wentzel-Kramers-Brillouin-Jeffreys (WKBJ) analysis (Bender \& Orszag 1978), where it corresponds to the eikonal equation for the leading-order term $S_{0}$ associated with the stability problem and evaluated at $y=y_{*}$. This leading-order term can be expanded in a Taylor series $S_{0}\left(y \rightarrow y_{*}\right)=\mathrm{i} \beta_{*} y+C\left(y-y_{*}\right)^{2}+$ h.o.t., and the requirement of a compact wave packet calls for the real part of $C$ to be negative. This latter condition can be expressed in terms of the symbol $f$ and leads to the so-called twist condition

$$
\operatorname{Im}\left\{\frac{\partial f}{\partial y_{*}} / \frac{\partial f}{\partial \beta_{*}}\right\}<0
$$

The symbol $f$ and the twist condition (3.1) yield a complete description of wave packet pseudomodes and relate them to their (approximate) eigenvalues $\lambda=f\left(y_{*}, \beta_{*}\right)$.

The theory of wave packet pseudomodes enables a local study of the stability at $y=$ $y_{*}$ while the global context of the flow field remains included in the analysis through the twist condition. Inhomogeneous flow directions (e.g. the wall-normal direction) can be treated in a similar manner as a normal wave ansatz can be applied to homogeneous directions. Therefore, dispersion relations with differential operators, such as (1.2), can be replaced by algebraic expressions of the form $f\left(y_{*}, \beta_{*}\right)=\lambda$, which allows a simpler analysis of the spectrum and yields explicit relations between (pseudo-)modes and flow parameters. The application of the theory of wave packet pseudomodes is promising whenever there exist modal solutions in the shape of wave packets at a sufficient distance from the boundaries. It turns out that eigensolutions of that character can often be predicted by this theory as accurately as by a numerical solution of the eigenvalue problem.

\subsection{Uniform modes $(N=0)$}

It is instructive to apply the theory of wave packet pseudomodes first to the case $N=0$, which describes uniform modes. For this class of modes, only perturbations $\hat{u}$ in the chordwise direction need to be considered; furthermore, modes of type A are not supported for $N=0$. The stability problem (1.2) reduces to the ordinary differential equation

$$
\left[\partial_{y}^{2}-V \partial_{y}+V^{\prime}-\gamma^{2}-\mathrm{i} \gamma \operatorname{Re} W\right] \hat{u}=-\mathrm{i} \operatorname{Re} \lambda \hat{u}
$$


with boundary conditions $\hat{u}(0)=\hat{u}(y \rightarrow \infty)=0$. The symbol of this eigenvalue problem (3.2) is given as

$$
f\left(y_{*}, \beta_{*}\right)=-\mathrm{i} \beta_{*}^{2}+\epsilon V\left(y_{*}\right) \beta_{*}+\mathrm{i} \epsilon^{2}\left[V^{\prime}\left(y_{*}\right)-\gamma^{2}\right]+\gamma W\left(y_{*}\right)
$$

with $\epsilon=1 / \sqrt{R e}$ (see also Obrist \& Schmid 2010 for a detailed discussion of uniform wave packet pseudomodes in the context of swept Hiemenz flow). To illustrate the mapping from the wave packet parameters $\left(y_{*}, \beta_{*}\right)$ to the approximate eigenvalues $\lambda$, we show the so-called 'symbol curves' $\lambda=f\left(y_{*},-\infty \cdots \infty\right)$ in figure 5. In addition, if we evaluate the twist condition (3.1) on these symbol curves, we determine regions in the complex eigenvalue plane where wave packet pseudomodes are supported. As can be seen in figure 5, wave packet pseudomodes exist nearly everywhere and for every wavenumber - except for parts of the boundary-layer region and for $y_{*} \rightarrow \infty$, i.e. where the base flow blends into a parallel free-stream flow. We also observe that the symbol curves cover most of the lower complex half-plane. This suggests that the wave packet pseudomodes in the free stream are part of a continuous spectrum which covers a large area in the lower half of the complex plane. The upper boundary of this area corresponds to the limiting symbol curve for $y_{*} \rightarrow \infty$,

$$
f\left(y_{*} \rightarrow \infty, \beta_{*}\right)=\gamma+\beta_{*} /(\epsilon \tan \varphi)-\mathrm{i}\left(\beta_{*}^{2}+\epsilon^{2} \gamma^{2}\right) .
$$

All other symbol curves (except for the symbol curves of the boundary layer) lie below this parabola. Since its apex lies above the limit (1.3b) for algebraic decay, it verifies the existence of a continuous spectrum of algebraically diverging modes. The eigenfunctions shown in figure 3 are part of this spectrum. If we choose $\lambda$ within this continuous spectrum, a singular value decomposition shows that the discretized eigenvalue problem (3.2) is satisfied up to machine precision, i.e. its smallest singular value $\sigma_{\min }$ is of the order of $10^{-15}$ (with respect to an appropriate energy norm). Therefore, these eigenvalues $\lambda$ are as accurate as actual eigenvalues, and the associated left singular vectors describe actual eigenmodes, not just pseudomodes.

\subsection{Higher-order modes $(N \geqslant 1)$}

For higher-order modes with $N \geqslant 1$ the generalized stability problem (1.2) constitutes a $2 \times 2$ system of equations. The symbol $\boldsymbol{F}$ for this eigenvalue problem is given by the expression

$$
\underbrace{\left[\begin{array}{ll}
f_{1} & f_{2} \\
f_{3} & f_{4}
\end{array}\right]}_{\boldsymbol{F}}\left[\begin{array}{l}
u \\
v
\end{array}\right]=\lambda\left[\begin{array}{l}
u \\
v
\end{array}\right]
$$

with

$$
\begin{gathered}
f_{1}=\gamma W+\epsilon V \beta_{*}-\mathrm{i}\left[\beta_{*}^{2}+\epsilon^{2} \gamma^{2}-\epsilon^{2}(N+1) V^{\prime}\right], \\
f_{2}=\mathrm{i} \epsilon^{2} V^{\prime \prime}, \\
f_{3}=-2 N\left(\epsilon^{3} V^{\prime} \beta_{*}-\mathrm{i} \epsilon^{4} V^{\prime \prime}\right) /\left(\beta_{*}^{2}+\epsilon^{2} \gamma^{2}\right), \\
f_{4}=\gamma W+\epsilon V \beta_{*}-\mathrm{i}\left[\beta_{*}^{2}+\epsilon^{2} \gamma^{2}-\epsilon^{2}(N-2) V^{\prime}\right] \\
-\left(\epsilon^{3} V^{\prime \prime} \beta_{*}-\epsilon^{2} \gamma W^{\prime \prime}-\mathrm{i} \epsilon^{4} N V^{\prime \prime \prime}\right) /\left(\beta_{*}^{2}+\epsilon^{2} \gamma^{2}\right) .
\end{gathered}
$$




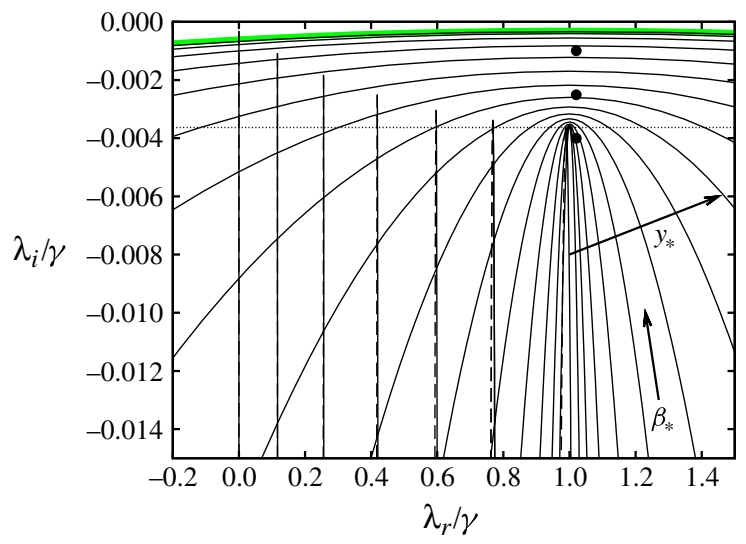

FIGURE 5. (Colour online available at journals.cambridge.org/flm) Symbol curves for uniform modes $(N=0)$ : narrow parabolas for $y_{*}$ in the boundary layer and wide parabolas in the free stream. The parabolas for the boundary layer are so narrow that they appear as vertical lines. Dashed lines indicate that the twist condition is not satisfied. The pale grey (green) parabola is the limiting symbol curve (3.4) for which the twist condition is also not satisfied. The dotted horizontal line indicates the limit $(1.3 b)$ for algebraically diverging modes of type $\mathrm{B}$. The dots indicate the eigenvalues of the three modes shown in figure 3.
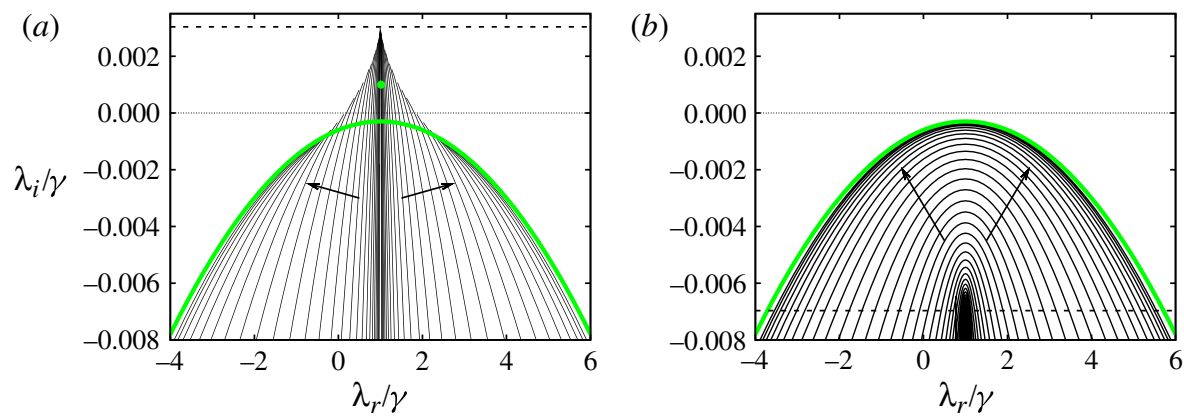

FIGURE 6. (Colour online) Symbol curves $f\left(y_{*},-\infty \cdots \infty\right)$ outside the boundary layer for $N=1$ and $4<y_{*}<\infty$ (the arrows indicate an increasing $y_{*}$ ): (a) $\lambda^{+}$-branch, $(b) \lambda^{-}$-branch. Only the sections of the symbol curves are shown for which the twist condition is satisfied. The pale grey (green) parabola is the limiting symbol curve for $y_{*} \rightarrow \infty$ (for which the twist condition is not satisfied). The dashed line in $(a)$ indicates the limit $(1.3 a)$ for algebraically diverging modes of type A. The dashed line in $(b)$ indicates the corresponding limit $(1.3 b)$ for modes of type B. The pale grey (green) dot shows the location of the (pseudo-)eigenvalue used in figure 7.

From this symbol, we can compute approximations to the eigenvalues $\lambda$ by solving the algebraic eigenvalue problem $(\boldsymbol{F}-\lambda \boldsymbol{I}) \boldsymbol{u}=0$. This gives

$$
\lambda^{ \pm}=\frac{1}{2}\left[\operatorname{tr}(\boldsymbol{F}) \pm \sqrt{\operatorname{tr}(\boldsymbol{F})^{2}-4 \operatorname{det}(\boldsymbol{F})}\right] .
$$

In contrast to the scalar eigenvalue problems for the uniform modes, we encounter two eigenvalue branches which we denote by $\lambda^{+}$and $\lambda^{-}$. The twist condition for the 
corresponding wave packet pseudomodes now reads

$$
\operatorname{Im}\left\{\frac{\partial}{\partial y_{*}}\left[\operatorname{det}(\boldsymbol{F})-\lambda^{ \pm} \operatorname{tr}(\boldsymbol{F})\right] / \frac{\partial}{\partial \beta_{*}}\left[\operatorname{det}(\boldsymbol{F})-\lambda^{ \pm} \operatorname{tr}(\boldsymbol{F})\right]\right\}<0 .
$$

The symbol curves for Görtler-Hämmerlin modes $(N=1)$ are shown in figure 6 . The individual symbol curves again have the shape of parabolas (see e.g. the $\lambda^{-}$-branch in figure $6 b$ ). For the $\lambda^{+}$-branch, the family of parabolas forms a peculiarly pointed structure (see figure $6 a$ ). Close to the apex of each parabola, where it overlaps with the symbol curves for smaller $y_{*}$, the twist condition (3.8) is not satisfied. Therefore, the symbol curves in figure 6(a) consist of just the 'legs' of the parabolas. There exists a unique map between the $\lambda^{+}$in this region and a parameter pair $\left(y_{*}, \beta_{*}\right)$. The boundary of the pointed region is the envelope of all symbol curves. For a scalar symbol $f\left(y_{*}, \beta_{*}\right)$, the envelope is then simply given by $\partial f / \partial y_{*}=0$, and we note that this equation is formally equivalent to the limiting value of the twist condition (3.1). For a vector-valued symbol $\boldsymbol{F}$, the envelope is given by

$$
\frac{\partial}{\partial y_{*}}\left[\operatorname{det}(\boldsymbol{F})-\lambda^{ \pm} \operatorname{tr}(\boldsymbol{F})\right]=0 .
$$

Therefore, the envelope(s) of the family of all symbol curves can also be understood as a boundary between regions in the eigenvalue plane where the twist condition is satisfied or violated.

The tip of the envelope for $\lambda^{+}$is at $\gamma+\mathrm{i}\left(1-\gamma^{2}\right) / R e$, which is consistent with the limit $(1.3 a)$ obtained for the classical swept Hiemenz flow. For $\gamma<1$, the symbol curves reach into the unstable half-plane, which indicates that there may exist unstable modes; nevertheless, we have not been able to find proper eigenmodes in this part of the spectrum. There exist, however, optimal pseudomodes which can be determined from a singular value decomposition of the linear operator in (1.2) (Schmid \& Henningson 2000). Figure 7 illustrates the evolution of such a pseudomode at $\lambda / \gamma=$ $1.017+0.001 \mathrm{i}$ for which the singular value decomposition yields a smallest singular value of approximately 0.026 . The initial growth of this perturbation is just a transient phenomenon. It is the result of a pile-up of energy as the wave packet is advected at high velocity toward the wall. After this period of transient growth, the perturbation decays to zero. We conclude that the symbol curves in the upper complex half-plane describe only pseudomodes but not proper eigenmodes. This is in contrast to the symbol curves in the lower half-plane, e.g. all symbol curves from the $\lambda^{-}$-branch. There we can find modal solutions which satisfy the eigenvalue problem (1.2) exactly. This is illustrated in figure 8 , which shows that the smallest singular value $\sigma_{\min }$ is near machine precision for $\lambda$ beneath the limiting symbol curve. In the vicinity of the limiting symbol curve, $\sigma_{\min }$ rises sharply, indicating that the corresponding solutions are pseudomodes rather than eigenmodes.

With respect to the algebraic modes, the two branches $\lambda^{ \pm}$correspond to the two asymptotic behaviours mentioned earlier (types A and B). The modes of the $\lambda^{+}$-branch can be identified by inspection with the asymptotic behaviour of type A, $\hat{v} \sim y^{-v-4}$. For $N=1$, all eigenvalues of the $\lambda^{+}$-branch satisfy condition (1.3a). Therefore, no algebraically diverging modes can be found on this branch for $N=1$. The $\lambda^{-}$-branch corresponds to modes of type B, i.e. $\hat{u} \sim y^{-v-1}$. As shown in figure $6(b)$, some of its eigenvalues lie above the bound (1.3b); consequently, algebraically diverging modes of type B exist. 

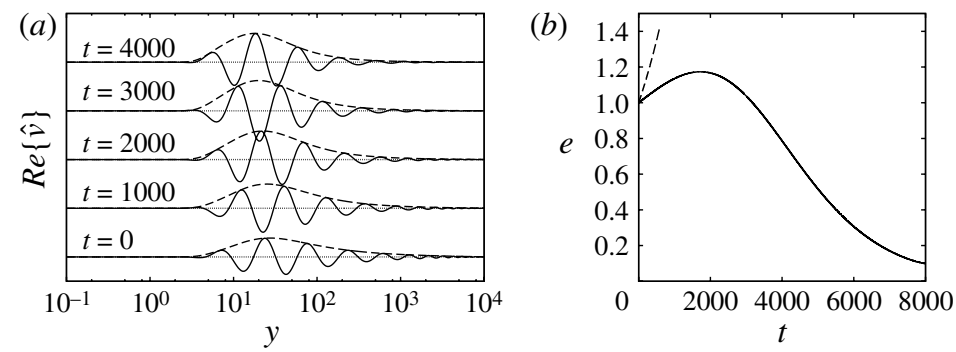

FIGURE 7. Evolution of an optimal pseudomode for $N=1$ and $R e=1000$ whose associated pseudoeigenvalue is located in the upper half-plane $(\lambda / \gamma=1.017+0.001 \mathrm{i}):(a)$ snapshots of the wall-normal velocity $\hat{v} ;(b)$ growth and decay of the pseudomode over time where $e=\int_{0}^{\infty}\left(|\hat{u}|^{2}+|\hat{v}|^{2}\right) \mathrm{d} y$ (the dashed line in $(b)$ indicates the growth according to $\left.\exp 2 \lambda_{i} t\right)$.

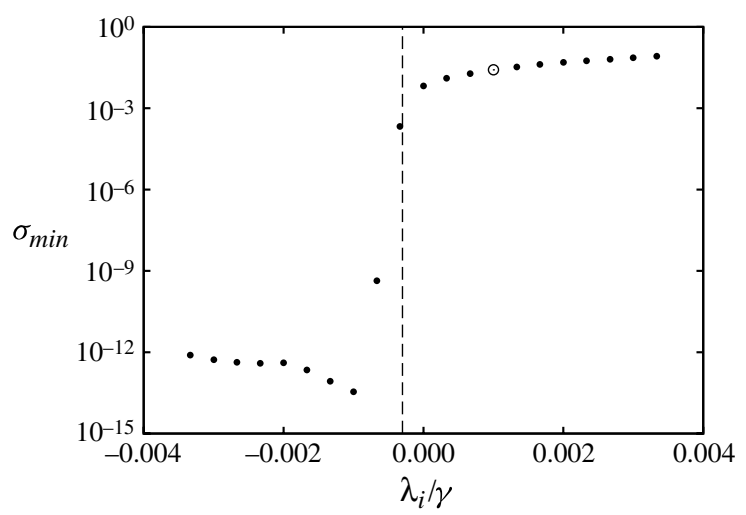

FIGURE 8. Smallest singular value $\sigma_{\min }$ (with respect to an appropriate energy norm) of the discretized eigenvalue problem (1.2) for $\lambda / \gamma=1.017+\mathrm{i} \lambda_{i} / \gamma$. The dashed line indicates the position of the limiting symbol curve and the open circle corresponds to the pseudomode shown in figure 7. The equations are discretized with a Chebyshev collocation method on 3000 Gauss-Lobatto points. The grid points are redistributed in the interval $\left[0,1.8 \times 10^{5}\right]$ with an algebraic grid stretching which places one half of the points in the interval $[0,2000]$.

The symbol curves for $N=2,3, \ldots$ produce a structure very similar to the symbol curves shown in figure $6(b)$. There exists a common limiting symbol curve for $y_{*} \rightarrow$ $\infty$, namely

$$
\left.\boldsymbol{F}\right|_{y_{*} \rightarrow \infty}=\left[\begin{array}{cc}
\xi & 0 \\
0 & \xi
\end{array}\right] \quad \text { with } \xi=\gamma+\beta_{*} /(\epsilon \tan \varphi)-\mathrm{i}\left(\beta_{*}^{2}+\epsilon^{2} \gamma^{2}\right) .
$$

Both eigenvalue branches converge toward the limiting symbol curve (3.4), which we have already found for $N=0$. This limiting symbol curve is valid for all $N$. Except for the $\lambda^{+}$-branch of $N=1$, the limiting symbol curve is identical to the envelope of all symbol curves (see figure 6). Therefore, algebraically diverging modes of type A exist for eigenvalues within the range

$$
\left(2-N-\gamma^{2}\right) / \operatorname{Re}<\lambda_{i}<-\left[\left(\lambda_{r}^{2}-2 \lambda_{r} \gamma\right) \tan ^{2} \varphi+\gamma^{2} \cos ^{2} \varphi\right] / \operatorname{Re},
$$




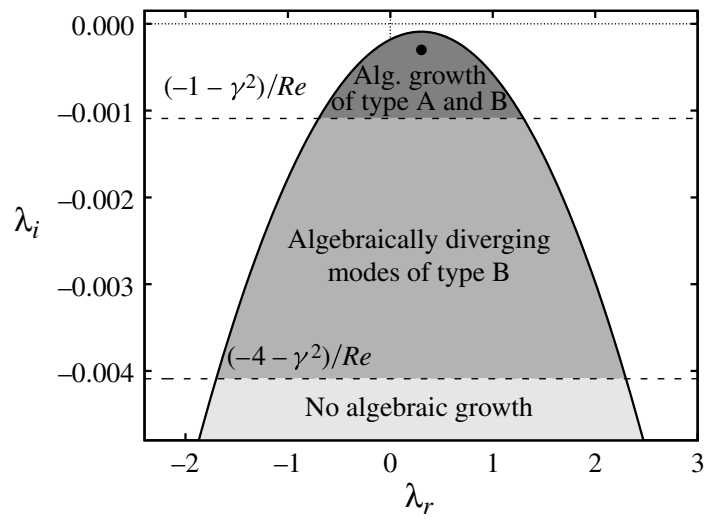

FIGURE 9. Shape of the continuous spectrum for algebraically diverging modes of order $N=3$ upstream of a bluff body as predicted by conditions (3.11) and (3.12). The dot indicates the eigenvalue for the mode shown in figure 4.

and for modes of type $\mathrm{B}$ we obtain algebraically diverging modes for

$$
\left(-1-N-\gamma^{2}\right) / \operatorname{Re}<\lambda_{i}<-\left[\left(\lambda_{r}^{2}-2 \lambda_{r} \gamma\right) \tan ^{2} \varphi+\gamma^{2} \cos ^{2} \varphi\right] / R e
$$

where the upper bounds describe the parabola of the limiting symbol curve (3.4). These relations are illustrated in figure 9 for $N=3$. Since the apex of the limiting symbol curves is at $-\gamma^{2} / \operatorname{Re}$ for all modes, we can determine the maximum algebraic growth rate for the modes upstream of a bluff body universally as

$$
\begin{aligned}
& \text { type } \mathrm{A}: N-2, \\
& \text { type } \mathrm{B}: N+1 .
\end{aligned}
$$

This remarkably simple result confirms our earlier observation that algebraically diverging modes of type A are only possible for $N \geqslant 3$ while type B modes can exhibit algebraic growth for all polynomial orders $N$.

\section{Concluding remarks}

The extension of the classical swept Hiemenz boundary layer to a base flow which includes the parallel flow upstream of a swept bluff body has provided new insight into the eigenvalue spectrum of the algebraic modes and revealed how these modes connect with the outer flow. They decay or grow algebraically only within the matching layer (stagnation-point flow), but decay superexponentially as soon as the base flow blends into a parallel external flow. For this reason, the boundary conditions at $y \rightarrow \infty$ can also be satisfied for modes which diverge algebraically within the matching layer. Despite this possibility, the algebraic growth rate remains bounded by (3.13). This suggests that studies of the linear stability of the swept Hiemenz flow should relax the free-stream boundary conditions to allow for diverging solutions in the free stream. Alternatively, the homogeneous boundary conditions in the free stream can be maintained by introducing the new dependent variables $(\bar{u}, \bar{v})$ according to

$$
\hat{u}=y^{N+1} \bar{u} \quad \text { and } \quad \hat{v}=y^{N-2} \bar{v} .
$$

These new variables satisfy homogeneous boundary conditions up to the algebraic growth rates given in (3.13). However, this change of variables restricts the eigenvalue 
spectrum only to $\lambda_{i}<-\left[\lambda_{r}^{2}-2 \lambda_{r} \gamma+2 \gamma^{2}\right] / \operatorname{Re}$ (see (3.11) and (3.12)). Therefore, we must additionally reject all modes which lie to the left or right of the limiting parabola (3.4).

Algebraically diverging modes could be of significance for the receptivity of leading-edge boundary layers. For plane stagnation-point flow, it has been shown by Dhanak \& Stuart (1995) that algebraically diverging modes can be used for connecting free-stream disturbances with boundary-layer modes. For the swept leading-edge boundary layer, receptivity mechanisms could be analysed along the lines of Zaki \& Durbin (2005), who investigated the interaction between free-stream disturbances and the flat-plate boundary layer in the context of bypass transition. To this end, free-stream disturbances were described by modes from the continuous spectrum. We envision a similar role for the algebraic modes because they are equivalent in character to the continuous modes in the Blasius boundary layer (see $\S 5$ in Obrist \& Schmid 2010). Such a receptivity analysis would use the algebraic modes for an expansion of free-stream disturbances which will connect these disturbances to the boundary layer. An expansion of travelling wave packets in the free stream to a series of wave packet pseudomodes has already been discussed by Obrist \& Schmid (2010). The application of this concept to algebraically diverging modes is straightforward.

Besides its potential significance for boundary-layer receptivity, the present study is intended to further contribute to the accurate and complete description of the spectral signature of flow around bluff bodies and to provide tools (in the form of wave packet pseudomodal analysis) to accomplish this goal.

\section{REFERENCES}

Bender, C. M. \& ORSZAG, S. A. 1978 Advanced Mathematical Methods for Scientists and Engineers. McGraw-Hill.

DhanaK, M. R. \& Stuart, J. T. 1995 Distortion of the stagnation-point flow due to cross-stream vorticity in the external flow. Phil. Trans. R. Soc. Lond. A 352, 443-452.

GÖRTLER, H. 1955 Dreidimensionale Instabilität der ebenen Staupunktströmung gegenüber wirbelartigen Störungen. In 50 Jahre Grenzschichtforschung (ed. H. Görtler \& W. Tollmien). p. 304. Vieweg.

Hall, P., Malik, M. R. \& Poll, D. I. A. 1984 On the stability of an infinite swept attachment line boundary layer. Proc. R. Soc. Lond. A 395, 229-245.

HÄMmERLIN, G. 1955 Zur Instabilitätstheorie der ebenen Staupunktströmung. In 50 Jahre Grenzschichtforschung (ed. H. Görtler \& W. Tollmien), p. 315. Vieweg.

HiemenZ, K. 1911 Die Grenzschicht an einem in den gleichförmigen Flüssigkeitsstrom eingetauchten geraden Kreiszylinder. PhD thesis, Göttingen.

OBRIST, D. \& SChMID, P. J. 2003 On the linear stability of swept attachment-line boundary-layer flow. Part 1. Spectrum and asymptotic behaviour. J. Fluid Mech. 493, 1-29.

OBRist, D. \& Schmid, P. J. 2010 Algebraically decaying modes and wave packet pseudomodes in swept Hiemenz flow. J. Fluid Mech. 643, 309-331.

Schmid, P. J. \& Henningson, D. S. 2000 Stability and Transition in Shear Flows. Springer.

TRefethen, L. N. 2005 Wave packet pseudomodes of variable coefficient differential operators. Proc. R. Soc. A 461 (2062), 3099-3122.

Trefethen, L. N. \& Embree, M. 2005 Spectra and Pseudospectra: The Behavior of Nonnormal Matrices and Operators. Princeton University Press.

ZAKI, T. A. \& DURbin, P. A. 2005 Mode interaction and the bypass route to transition. J. Fluid Mech. 531, $85-111$. 